\title{
Development of Rapid Assays for Detection and Identification of Pathogenic Bacteria
}

\author{
Haiyan $L i^{1}$ and Sam F. Y. $L i^{1,2}$ \\ ${ }^{1}$ Department of Chemistry, National University of Singapore, 3 Science Drive 3, Singapore 117543, \\ ${ }^{2}$ NUS Environmental Research Institute, National University of Singapore, \#02-01, T-Lab Building, 5A \\ Engineering Drive 1, Singapore 117411 \\ Corresponding author's e-mail address: chmlifys@nus.edu.sg
}

\begin{abstract}
:
Bacterial contamination in water and food poses serious hazards worldwide and the rapid detection of pathogenic bacteria is critical to the prevention and identification of problems related to public health and safety. This study focuses on the development of rapid detection assays by using biosensors for rapid, sensitive, specific pathogens detection in water or complex food matrices. Quartz crystal microbalance biosensors with different bio-recognition elements, strategies for improved sensitivity by nanomaterials, sample preparations to eliminate interference were explored. The measurements in all biosensors were reproducible with relative standard deviation (RSD) less than 15\%. Non-cultural method for food sample preparation was built to rapidly and efficiently separate most of the interfering particles without sacrificing bacterial recovery. Furthermore, nanomaterials were utilized for improving sensitivity of biosensors. Gold nanoparticles (AuNPs) were synthesized and characterized for QCM signals amplification. Label-free QCM biosensors with different sensitivity and assay time based on self-assembled monolayers (SAMs) have been set up. A detection limit as low as $\sim 10^{2} \mathrm{CFU} / \mathrm{mL}$ for $E$. coli could be achieved when AuNPs were used and total assay time was less than $2 \mathrm{~h}$.
\end{abstract}

Key words: surface plasmon resonance, quartz crystal microbalance, rapid assays, pathogenic, bacteria

\section{Introduction}

As an important part of water or food safety and security, their quality is emphasized worldwide nowadays. Water quality control includes water condition measurements of biological, chemical, and physical parameters [1]. Among these parameters, microbiological risk is considered as a top priority in drinking water. Food safety is another issue which is causing growing concerns in recent years and microbiological analysis is an integral part of food quality control and the management of food chain [2]. Thus, rapid and reliable bacteriological monitoring is of utmost importance and necessary for advance warning of risk and the prevention of waterborne and foodborne diseases for public health. In this study, rapid detection of bacteria in food via quartz crystal microbalance (QCM) is demonstrated. Noncultural enrichment technologies were developed for sample preparation to remove the interferences while reducing the need for time-consuming cultural enrichments.

\section{Experimental}

Piezoelectric QCM is a very sensitive massmeasuring instrument, which consists of an ATcut quartz crystal disc that exhibits piezoelectric behavior and metal electrodes deposited on opposite faces for applying alternating electric fields [3]. When an electric field is applied, physical deformation of the crystal will be produced. The inverse piezoelectric effect induces a frequency shift in the crystals when there is a mass change on the modified electrode surface. The relation between mass and resonant frequency is given by the Sauerbrey equation [3]:

$$
\Delta f=-\frac{2 f^{2}}{A \sqrt{\rho_{q}^{\prime} \mu_{q}}} \Delta m
$$


where $f$ is the intrinsic resonant frequency, $\Delta f$ is the frequency change, $\Delta m$ is the mass change due to the surface deposition, $\rho_{q}$ is the density of quartz, $\mu_{q}$ is the shear modulus and $\mathrm{A}$ is the area of the quartz crystal. The resonance frequency changes on a quartz crystal are proportional to mass changes on the probe or transducer surface (e.g. gold surface). Thus, mass changes generated from biological binding reactions could be real-time monitored by measuring frequency shift. Due to its simplicity, cost effectiveness, label free and real-time monitoring function, $\mathrm{QCM}$ is a powerful tool for specific, sensitive, and rapid detection of bacteria when incorporated with highly specific bio-recognition elements.

Since nanomaterials can benefit the development of biosensors as signal amplifiers, a sensitive and label-free QCM with dissipation (QCM-D) biosensor with antibodies and 2- step signal amplification was developed for detection of E. coli and L. pneumophia (Figure 1).

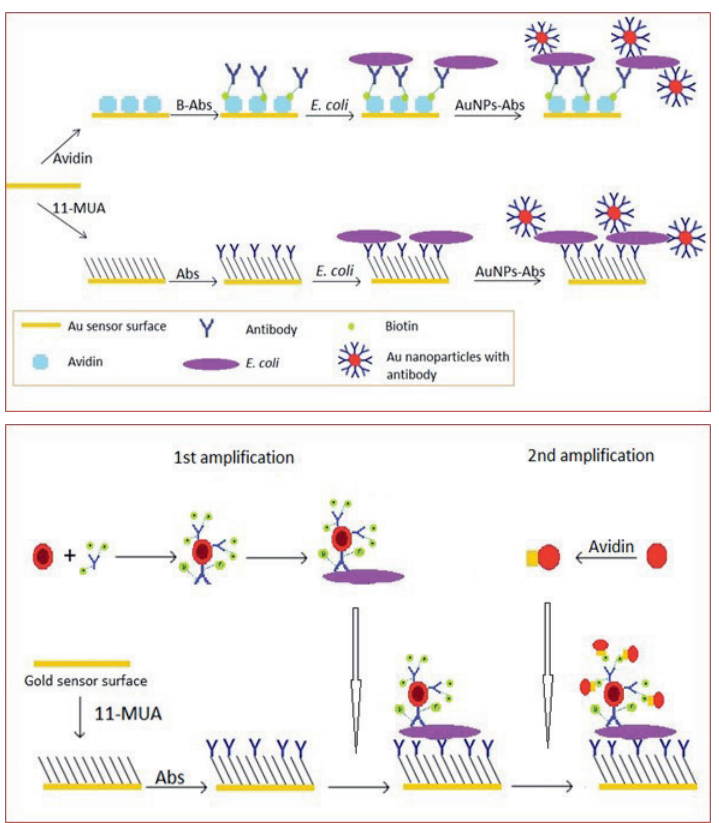

Figure 1 Schematic of the step-by-step fabrication for the E. coli QCM sensor with 1 amplifier $(A)$ and 2 amplifiers (B).

\section{Results and Discussion}

Different strategies for improving the sensitivity based on bio-recognition elements immobilization and signal amplification was addressed and optimized. Gold nanoparticles (AuNPs) were synthesized, characterized and applied for signal amplification of these biosensors. QCM-D biosensors with different detection limit and assay time are presented in this section (Figure 2).
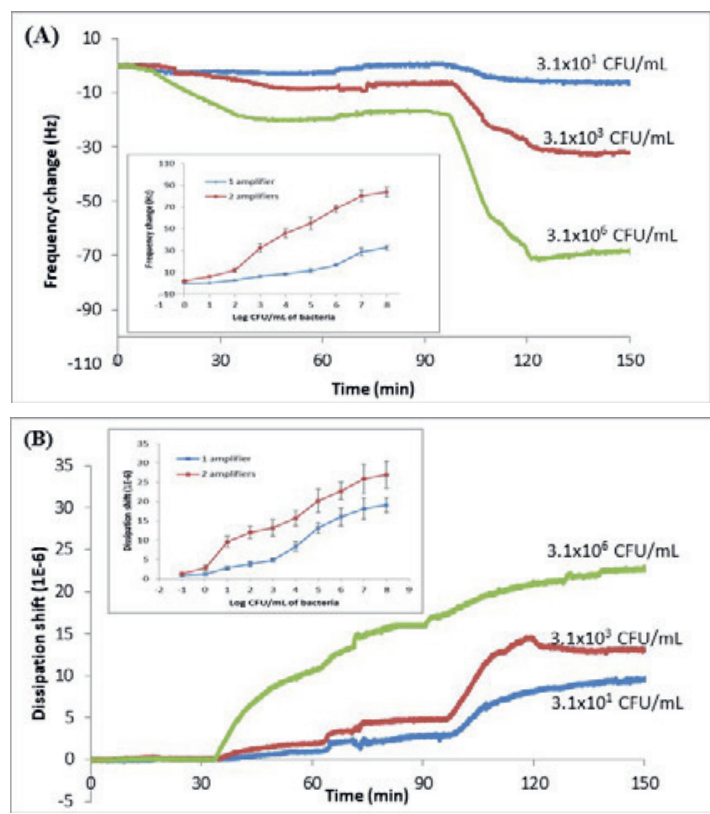

Figure 2 On-line responses (3rd overtone) of frequency $(\Delta f)(A)$ and dissipation $(\Delta D)$ (B) from $Q C M-D$ biosensor with 2 amplifiers for $L$. pneumophila detection at different concentrations. Insets: the relationships between $\Delta f$ and $\Delta D$ as a function of cell concentration. Error bars are standard deviation from triplicates trial.

\section{Conclusion}

The results showed that when AuNPs were used to modify the sensor surface and to amplify the signals, QCM immunosensors showed good detection sensitivity, i.e. detection limit of $\sim 10^{2} \mathrm{CFU} / \mathrm{mL}$ for $E$. coli in less than $1 \mathrm{~h}$ could be achieved. Though the modification process on the sensor surface still involved several steps, the immobilization process could be readily replaced by easy regeneration of antibodies immobilized sensor surface.

\section{References}

[1] R. Diaz-Chavez, G. Berndes, D. Neary, A.E. Neto, M. Fall, Water quality assessment of bioenergy production, Biofuels, Bioprod. Biorefin., 5 (2011) 445463.

[2] D. De Medici, T. Kuchta, R. Knutsson, A. Angelov, B. Auricchio, M. Barbanera, C. Diaz-Amigo, A. Fiore, E. Kudirkiene, A. Hohl, D.H. Tomic, V. Gotcheva, B. Popping, E. Prukner-Radovcic, S. Scaramaglia, P. Siekel, K.A. To, M. Wagner, Rapid methods for quality assurance of foods: the next decade with polymerase chain reaction (PCR)-based food monitoring, Food Anal. Meth., 8 (2015) 255-271.

[3] G. Sauerbrey, Verwendung von schwingquarzen zur Wägung dünner schichten und zur mikrowägung, Z. Phys., 155 (1959) 206-222. 